\title{
NON-UNICITÉ DU PROBLÈME DE CAUCHY POUR DES OPÉRATEURS DIFFÉRENTIELS QUASI-HOMOGÈNES
}

\author{
KHALGUI-OUNAÏES HELLA
}

Reçu le 19 juin 2000

\begin{abstract}
Nous démontrons que si $P$ est un opérateur différentiel quasi-homogène d'ordre $m$ sur une partie ouverte $\Omega$ de $\mathbb{R}^{n}$, à coefficients de classe $C^{\infty}$, tel que la $m$-partie principale est à coefficients réels ; et que $x_{0} \in \Omega, S=\left\{x \in \Omega: \phi(x)=\phi\left(x_{0}\right)\right\}$ est une hypersurface non caractéristique en $x_{0}$ et strictement non pseudoconvexe avec $\left\{\left\{p_{m}, \phi\right\}, \phi\right\}\left(x_{0}, \xi_{0}\right) \neq 0$ et $d_{q} p_{m}\left(x_{0}, \xi_{0}\right) \neq 0$, alors $P$ n'a pas l'unicité de Cauchy par rapport à $S$.
\end{abstract}

Classification 2000 des Sujets Mathématiques: 35A07.

1. Introduction. Soit $\Omega$ une partie ouverte de $\mathbb{R}^{n}$ et $S$ une hypersurface de $\Omega$, passant par un point $x_{0}$, définie par

$$
S=\left\{x \in \Omega: \phi(x)-\phi\left(x_{0}\right)=0\right\},
$$

où $\phi$ est une fonction réelle de classe $C^{\infty}$ vérifiant $d \phi\left(x_{0}\right) \neq 0$.

DÉfinition 1.1. Soit $P$ un opérateur différentiel défini sur $\Omega$, on dit que $P$ n'a pas l'unicité de Cauchy par rapport à $S$ s'il existe un voisinage $V$ de $x_{0}$ dans $\Omega$, des fonctions $a$ et $u \neq 0$, de classe $C^{\infty}$ sur $V$ tel que supp $a \subset\left\{x \in \Omega: \phi(x) \leq \phi\left(x_{0}\right)\right\}$,

$$
\begin{gathered}
V \cap \operatorname{supp} u=\left\{x \in \Omega: \phi(x) \leq \phi\left(x_{0}\right)\right\} \cap V, \\
P u+a u=0 \text { dans } V .
\end{gathered}
$$

Alinhac [1] a donné des résultats de non-unicité pour des opérateurs du type

$$
p\left(x, t, \sigma^{q} \xi, \sigma \tau\right)=\sigma^{m} p_{m}(x, t, \xi, \tau)+\sigma^{m-k} p_{m-k}(x, t, \xi, \tau)+\cdots,
$$

où $x \in \mathbb{R}^{n-1}, t \in \mathbb{R}, q \geq 1$.

Dans ce papier, nous donnons des conditions suffisantes de non-unicité du problème de Cauchy pour des opérateurs différentiels quasi-homogènes, réels, à coefficients de classe $C^{\infty}$.

Les techniques utilisées dans ce travail sont rattachées aux constructions de l'optique géométrique qui sont développées dans les travaux de Plis [4], Hörmander [2] et Alinhac [1]. 
2. Notation et définitions. Soit $\Omega$ une partie ouverte de $\mathbb{R}^{n}$ et soit $(x, \xi) \in T^{*}(\Omega)$, $x=\left(x_{1}, \ldots, x_{n}\right)$.

Soit $m=\left(m_{1}, \ldots, m_{n}\right)$ un multi-indice tel que

$$
0<m_{1} \leq \cdots \leq m_{q-1}<m_{q}=\cdots=m_{n},
$$

et $\alpha=\left(\alpha_{1}, \ldots, \alpha_{n}\right) \in \mathbb{N}^{n}$.

On note

$$
\begin{gathered}
|\alpha: m|=\alpha_{1} m_{1}^{-1}+\alpha_{2} m_{2}^{-1}+\cdots+\alpha_{n} m_{n}^{-1} \\
D^{\alpha}=D_{1}^{\alpha_{1}} \cdots D_{n}^{\alpha_{n}} \quad \text { avec } D_{j}=\frac{1}{i} \frac{\partial}{\partial x_{j}}, \quad \nabla_{q}=\left(0, \ldots, 0, \frac{\partial}{\partial x_{q}}, \ldots, \frac{\partial}{\partial x_{n}}\right) .
\end{gathered}
$$

DÉfinition 2.1. On dit que $P$ est un opérateur différentiel quasi-homogène d'ordre $m \operatorname{sur} \Omega$ si

$$
P(x, D)=\sum_{|\alpha: m| \leq 1} a_{\alpha}(x) D^{\alpha} .
$$

La $m$-partie principale de $P$ est l'opérateur

$$
P_{m}(x, D)=\sum_{|\alpha: m|=1} a_{\alpha}(x) D^{\alpha}
$$

DÉfinition 2.2. Soient $f$ et $g$ deux fonctions de classe $C^{\infty}$ sur $T^{*} \Omega$. On désigne par $\{f, g\}$ le crochet de Poisson quasi-homogène de $f, g$ défini par

$$
\{f, g\}=\sum_{j=q}^{n}\left(\frac{\partial f}{\partial \xi_{j}} \frac{\partial g}{\partial x_{j}}-\frac{\partial f}{\partial x_{j}} \frac{\partial g}{\partial \xi_{j}}\right) .
$$

DÉFINITION 2.3. Soit $x_{0} \in \Omega$ et $\phi$ une fonction de classe $C^{\infty}$ dans $\Omega$ telle que $\nabla_{q} \phi\left(x_{0}\right) \neq 0$. L'hypersurface $S=\left\{x \in \Omega: \phi(x)=\phi\left(x_{0}\right)\right\}$ est dite strictement non pseudoconvexe au sens quasi-homogène par rapport aux bicaractéristiques de $P$ issues de $x_{0}$, si elle est non caractéristique et vérifie la condition suivante :

$$
\exists \xi_{0} \in \mathbb{R}^{n}-\{0\}: p_{m}\left(x_{0}, \xi_{0}\right)=H_{p_{m}} \phi\left(x_{0}, \xi_{0}\right)=0, \quad H_{p_{m}}^{2} \phi\left(x_{0}, \xi_{0}\right)<0,
$$

avec $H_{p_{m}} \phi=\left\{p_{m}, \phi\right\}$ et $H_{p_{m}}^{2} \phi=\left\{p_{m},\left\{p_{m}, \phi\right\}\right\}$.

\section{3. Énoncé du théorème}

THÉORÈme 3.1. Soit $P$ un opérateur différentiel quasi-homogène d'ordre $m$, à coefficients de classe $C^{\infty}$ sur une partie ouverte $\Omega$ de $\mathbb{R}^{n}$, tel que la m-partie principale est à coefficients réels. Soit $x_{0} \in \Omega$ et

$$
S=\left\{x \in \Omega: \phi(x)=\phi\left(x_{0}\right)\right\}
$$

une hypersurface non caractéristique en $x_{0}$ et strictement non pseudoconvexe au sens quasi-homogène par rapport aux bicaractéristiques de $P$ issues de $x_{0}$. Pour $\xi_{0}$ vérifiant (2.6), on suppose que

(i) $\left\{\left\{p_{m}, \phi\right\}, \phi\right\}\left(x_{0}, \xi_{0}\right) \neq 0$; 
(ii) $d_{q} p_{m}\left(x_{0}, \xi_{0}\right) \neq 0$ où $d_{q} p_{m}=\left(0, \ldots, 0, d_{\xi_{q}} p_{m}, \ldots, d_{\xi_{n}} p_{m}\right)$.

Alors il existe un voisinage $W$ de $x_{0}$ et deux fonctions a et $u \neq 0$, de classe $C^{\infty}$ sur $W$, s'annulant dans

$$
\left\{x \in W: \phi(x)>\phi\left(x_{0}\right)\right\}
$$

et vérifiant

$$
P u+a u=0, \quad x_{0} \in \operatorname{supp} u .
$$

EXEMPLE 3.2. Soit $P$ l'opérateur différentiel quasi-homogène défini sur $\mathbb{R}^{5}$, d'ordre $m=(1,2,4,4,4)$, de symbole

$$
\begin{aligned}
& p\left(y_{1}, y_{2}, x_{1}, x_{2}, t, \eta_{1}, \eta_{2}, \xi_{1}, \xi_{2}, \tau\right) \\
& \quad=\tau^{4}+\tau^{2}\left[(1+t) \xi_{2}^{2}+\xi_{1}^{2}\right]+(1+t)\left[(1+t) \eta_{2}^{2}-\xi_{1}^{2} \xi_{2}^{2}+t^{3} \eta_{1}\right]+x_{1} \xi_{2}^{3} .
\end{aligned}
$$

Pour $x_{0}=(0,0,0) \in \mathbb{R}^{2} \times \mathbb{R}^{2} \times \mathbb{R}, S=\left\{(y, x, t) \in \mathbb{R}^{5}: t=0\right\}$ et $\xi_{0}=(2,1,1,1,0)$, l'opérateur $P$ vérifie les hypothèses du théorème 3.1, il en résulte que $P$ n'a pas l'unicité de Cauchy par rapport à $S$.

4. Preuve du théorème 3.1. Toutes les hypothèses étant invariantes par changement de coordonnées respectant les coordonnées quasi-homogènes, on peut donc se ramener aux variables $(y, x, t) \in \mathbb{R}^{q-1} \times \mathbb{R}^{n-q} \times \mathbb{R}$, dans un voisinage $V$ de $x_{0}=(0,0,0)$, avec

$$
S=\{(y, x, t) \in V: t=0\}, \quad P=P\left(y, x, t, D_{y}, D_{x}, D_{t}\right) .
$$

On pose $s=\lambda(t-\delta)$ avec $\lambda=\delta^{-\theta}, \delta>0$ et $\theta>1$. L'opérateur $P$ s'écrit alors dans les coordonnèes $(y, x, s)$,

$$
P\left(y, x, t, D_{y}, D_{x}, D_{t}\right)=P\left(y, x, \delta+\frac{s}{\lambda}, D_{y}, D_{x}, \lambda D_{s}\right) .
$$

Prenons la solution $u$ sous la forme

$$
\begin{aligned}
u\left(y, x, \delta+\frac{s}{\lambda}\right)= & e^{i\left(\sum_{j=1}^{q-1} \sigma^{1 / m_{j}} \eta_{j} y_{j}+\sigma^{1 / m_{n}}(\tilde{\xi}(y, x, \delta)+(s / \lambda) \tau(y, x, \delta))\right)} \\
& \times e^{v \varphi(y, x, \delta, s)} e^{-\gamma(y, x, \delta)} w(y, x, \delta, s) .
\end{aligned}
$$

On définit un opérateur $\tilde{P}$ par $P u / u=\tilde{P} w / w$ avec

$$
\begin{aligned}
\tilde{P}\left(y, x, \delta, s, D_{y}, D_{x}, D_{s}\right) & \\
= & P\left(y, x, \delta+\frac{s}{\lambda},\left(\sigma^{1 / m j} \eta_{j}+\sigma^{1 / m_{n}} \tilde{\xi}+\sigma^{1 / m_{n}} \frac{s}{\lambda} \nabla_{j} \tau+\frac{\nu}{i} \nabla_{j} \varphi-\frac{1}{i} \nabla_{j} \gamma+D_{y_{j}}\right)_{1 \leq j \leq q-1},\right. \\
& \left.\sigma^{1 / m_{n}} \nabla_{x} \tilde{\xi}+\sigma^{1 / m_{n}} \frac{s}{\lambda} \nabla_{x} \tau+\frac{\nu}{i} \nabla_{x} \varphi-\frac{1}{i} \nabla_{x} \gamma+D_{x}, \sigma^{1 / m_{n}} \tau+\frac{1}{i} \lambda \nu \varphi_{s}^{\prime}+\lambda D_{s}\right),
\end{aligned}
$$

où $\nabla_{x}$ désigne le vecteur gradient par rapport à la variable $x$ et $\nabla_{j}$ désigne la dérivée par rapport à la variable $y_{j}$. 


\subsection{Choix de $\eta, \tilde{\xi}, \tau$}

LEMmE 4.1. Soit $p_{m}$ le $m$-symbole principal de $P ; \zeta_{0}=\left(\eta_{0}, \xi_{0}, \tau_{0}\right)$ satisfaisant les hypothèses du théorème 3.1. Alors on peut trouver un voisinage de l'origine dans $\mathbb{R}^{q-1} \times$ $\mathbb{R}^{n-q} \times \mathbb{R}$, des fonctions $\eta(y, x, t)=\left(\eta_{1}, \eta_{2}, \ldots, \eta_{q-1}\right)(y, x, t), \xi(y, x, t)=\left(\xi_{1}, \xi_{2}, \ldots\right.$, $\left.\xi_{q-1}\right)(y, x, t), \tau(y, x, t)$ et $\delta_{0}>0$ tels que pour tout $0<\delta<\delta_{0}$ et $(y, x)$ près de l'origine dans $\mathbb{R}^{q-1} \times \mathbb{R}^{n-q}$ on $a$

$$
\begin{gathered}
p_{m}\left(y, x, \delta, \eta, \nabla_{x} \xi, \tau\right)=\frac{\partial p_{m}}{\partial \tau}\left(y, x, \delta, \eta, \nabla_{x} \xi, \tau\right)=0, \\
\left(\eta(0), \nabla_{x} \xi(0), \tau(0)\right)=\zeta_{0} .
\end{gathered}
$$

PReUVE. On prend pour tout $(y, x, t), \eta(y, x, t)=\eta_{0}$ c'est-à-dire $\eta_{j}=\eta_{0 j}$.

D’après les hypothèses (2.6) et le (i) du théorème 3.1 , on a

$$
\frac{\partial p_{m}}{\partial \tau}\left(0, \zeta_{0}\right)=0, \quad \frac{\partial^{2} p_{m}}{\partial \tau^{2}}\left(0, \zeta_{0}\right) \neq 0,
$$

il résulte d'après le théorème des fonctions implicites qu'il existe une fonction $q$ de classe $C^{\infty}$,

$$
q: V\left(0, \eta_{0}, \xi_{0}\right) \longrightarrow V\left(\tau_{0}\right) \subset \mathbb{R}, \quad(y, x, t, \eta, \xi) \longmapsto q(y, x, t, \eta, \xi),
$$

vérifiant

$$
q\left(0, \eta_{0}, \xi_{0}\right)=\tau_{0}, \quad \frac{\partial p_{m}}{\partial \tau}(y, x, t, \eta, \xi, q(y, x, t, \eta, \xi))=0
$$

On note

$$
F(y, x, t, \eta, \xi)=p_{m}(y, x, t, \eta, \xi, q(y, x, t, \eta, \xi)),
$$

on a alors $F\left(0,0,0, \eta_{0}, \xi_{0}\right)=p_{m}\left(0,0,0, \eta_{0}, \xi_{0}, \tau_{0}\right)=0$ et pour tout $1 \leq j \leq n-q$,

$$
\begin{aligned}
\frac{\partial F}{\partial \xi_{j}}(y, x, t, \eta, \xi)= & \frac{\partial p_{m}}{\partial \xi_{j}}(y, x, t, \eta, \xi, q(y, x, t, \eta, \xi)) \\
& +\frac{\partial q}{\partial \xi_{j}}(y, x, t, \eta, \xi) \frac{\partial p_{m}}{\partial \tau}(y, x, t, \eta, \xi, q(y, x, t, \eta, \xi)) .
\end{aligned}
$$

Ainsi d'après l'hypothèse (ii) du théorème 3.1, on déduit que

$$
d_{\xi} F\left(0,0,0, \eta_{0}, \xi_{0}\right)=d_{\xi} p_{m}\left(0,0,0, \eta_{0}, \xi_{0}, \tau_{0}\right) \neq 0 .
$$

Il résulte du théorème d'Hamilton-Jacobi que l'équation

$$
F\left(y, x, t, \eta, \nabla_{x} \Psi(y, x, t, \eta)\right)=0, \quad \nabla_{x} \Psi\left(0,0,0, \eta_{0}\right)=\xi_{0},
$$

admet une solution $C^{\infty}, \Psi(y, x, t, \eta),(y, x, t)$ près de l'origine, $\eta$ voisin de $\eta_{0}$. On pose

$$
\xi(y, x, t)=\Psi\left(y, x, t, \eta_{0}\right), \quad \tau(y, x, t)=q\left(y, x, t, \eta_{0}, \nabla_{x} \xi(y, x, t)\right) .
$$


On choisit $\delta_{0}>0$ tel que pour tout $\delta, 0<\delta<\delta_{0}$, on a $(y, x, \delta)$ voisin de l'origine. Ceci achève la démonstration du lemme 4.1 .

On prend

$$
\begin{gathered}
\eta=\eta_{0}, \\
\tau(y, x, \delta)=q\left(y, x, \delta, \eta_{0}, \nabla_{x} \xi(y, x, \delta)\right), \\
\tilde{\xi}(y, x, \delta)=\xi(y, x, \delta)+\frac{c \cdot x}{\lambda},
\end{gathered}
$$

où $c$ est un vecteur constant de $\mathbb{R}^{n-q}$ qu'on fixera ultérieurement.

LEMME 4.2. Notons $\zeta_{0}=\left(\eta_{0}, \xi_{0}, \tau_{0}\right)$ et $\phi(y, x, t)=t$, on a la relation

$$
\left\{p_{m},\left\{p_{m}, \phi\right\}\right\}\left(0, \zeta_{0}\right)=-\left(p_{m}\right)_{\tau \tau}^{\prime \prime}\left(p_{m t}^{\prime}+\sum_{j=1}^{n-q} \frac{\partial p_{m}}{\partial \xi_{j}} \frac{\partial \tau}{\partial x_{j}}\right)\left(0, \zeta_{0}\right) .
$$

PReUVe. Dans toute cette preuve on écrira $p$ à la place de $p_{m}$. On a

$$
\{p, \phi\}=p_{\tau}^{\prime}, \quad\{p,\{p, \phi\}\}=\sum_{j=1}^{n-q}\left(p_{\xi_{j}}^{\prime} p_{\tau x_{j}}^{\prime \prime}-p_{x_{j}}^{\prime} p_{\tau \xi_{j}}^{\prime \prime}\right)+p_{\tau}^{\prime} p_{\tau t}^{\prime \prime}-p_{t}^{\prime} p_{\tau \tau}^{\prime \prime}
$$

D'après (4.5) on obtient pour tout $1 \leq j \leq n-q$

$$
p_{x_{j}}^{\prime}+\sum_{k=1}^{n-q} p_{\xi_{k}}^{\prime} \xi_{x_{j} x_{k}}^{\prime \prime}+p_{\tau}^{\prime} \tau_{x_{j}}^{\prime}=0
$$

d'où

$$
\begin{gathered}
p_{x_{j}}^{\prime}=-\sum_{k=1}^{n-q} p_{\xi_{k}}^{\prime} \xi_{x_{j} x_{k}}^{\prime \prime}-p_{\tau}^{\prime} \tau_{x_{j}}^{\prime}, \\
p_{\tau x_{j}}^{\prime \prime}+\sum_{k=1}^{n-q} p_{\tau \xi_{k}}^{\prime \prime} \xi_{x_{j} x_{k}}^{\prime \prime}+p_{\tau \tau}^{\prime \prime} \tau_{x_{j}}^{\prime}=0,
\end{gathered}
$$

d'où

$$
p_{\tau x_{j}}^{\prime \prime}=-\sum_{k=1}^{n-q} p_{\tau \xi_{k}}^{\prime \prime} \xi_{x_{j} x_{k}}^{\prime \prime}-p_{\tau \tau}^{\prime \prime} \tau_{x_{j}}^{\prime}
$$

par suite

$$
\begin{aligned}
\{p,\{p, \phi\}\}= & -\sum_{j, k=1}^{n-q} p_{\xi_{j}}^{\prime} p_{\tau \xi_{k}}^{\prime \prime} \xi_{x_{k} x_{j}}^{\prime \prime}-\sum_{j=1}^{n-q} p_{\xi_{j}}^{\prime} p_{\tau \tau}^{\prime \prime} \tau_{x_{j}}^{\prime} \\
& +\sum_{j, k=1}^{n-q} p_{\tau \xi_{j}}^{\prime \prime} p_{\xi_{k}}^{\prime} \xi_{x_{j} x_{k}}^{\prime \prime}+\sum_{j=1}^{n-q} p_{\tau \xi_{j}}^{\prime \prime} p_{\tau}^{\prime} \tau_{x_{j}}^{\prime}+p_{\tau}^{\prime} p_{\tau t}^{\prime \prime}-p_{t}^{\prime} p_{\tau \tau}^{\prime \prime}
\end{aligned}
$$

On en déduit que $\{p,\{p, \phi\}\}\left(0, \zeta_{0}\right)=-p_{\tau \tau}^{\prime \prime}\left(\sum_{j=1}^{n-q} p_{\xi_{j}}^{\prime} \tau_{x_{j}}^{\prime}+p_{t}^{\prime}\right)\left(0, \zeta_{0}\right)$. 


\subsection{Choix de $\varphi$}

LEMME 4.3. Il existe c dans $\mathbb{R}^{n-q}$ tel que

$$
p_{t}^{\prime}\left(0, \zeta_{0}\right)+\sum_{j=1}^{n-q}\left(\tau_{x_{j}}^{\prime}-c_{j}\right) p_{\xi_{j}}^{\prime}\left(0, \zeta_{0}\right)=0
$$

et il existe une fonction $\varphi(y, x, \delta, s)$ de classe $C^{\infty}$ dans $\left.V_{0} \times\right] 0, \delta_{0}[\times]-s_{0}, s_{0}\left[\right.$, où $V_{0}$ est un voisinage de $(0,0)$ dans $\mathbb{R}^{q-1} \times \mathbb{R}^{n-q}$ tels que

$$
p_{m}\left(y, x, \delta+\frac{s}{\lambda}, \eta, \nabla_{x} \tilde{\xi}+\frac{s}{\lambda} \nabla_{x} \tau, \tau+\sigma^{-1 / m_{n}} \lambda \nu D_{s} \varphi\right)=0,
$$

avec

$$
\operatorname{Re} \varphi(y, x, \delta, s)=\alpha(y, x, \delta) s+\beta(y, x, \delta, s) s^{2},
$$

où $\alpha$ et $\beta$ sont des fonctions de classe $C^{\infty}$ sur $\left.V_{0} \times\right] 0, \delta_{0}[\times]-s_{0}, s_{0}[$,

$$
\alpha(0,0,0)<0, \quad \beta(0,0,0,0)<0 .
$$

Preuve. Dans cette preuve on écrira $p$ à la place de $p_{m}$. Posons

$$
G(y, x, \delta, s, z)=\delta^{-\theta} p\left(y, x, \delta+\frac{s}{\lambda}, \eta, \nabla_{x} \tilde{\xi}+\frac{s}{\lambda} \nabla_{x} \tau, \tau+\delta^{\theta / 2} z\right)
$$

On a

$$
\nabla_{x} \tilde{\xi}=\nabla_{x} \xi+\frac{c}{\lambda}=\nabla_{x} \xi+c \delta^{\theta} .
$$

On voudrait trouver $z_{0} \in \mathbb{C}$ tel que

$$
\begin{aligned}
G\left(0,0,0,0, z_{0}\right) & =0, \\
\frac{\partial G}{\partial z}\left(0,0,0,0, z_{0}\right) & \neq 0 .
\end{aligned}
$$

Pour cela on pose $X=\left(y, x, \delta+s \delta^{\theta}, \eta, \nabla_{x} \xi+c \delta^{\theta}+s \delta^{\theta} \nabla_{x} \tau, \tau\right)$ et on applique la formule de Taylor à $p$ au point $X$ jusqu'à l'ordre 2 ; on obtient

$$
G(y, x, \delta, s, z)=\delta^{-\theta} p(X)+\delta^{-\theta / 2} z p_{\tau}^{\prime}(X)+\frac{z^{2}}{2} p_{\tau \tau}^{\prime \prime}(X)+O\left(\delta^{\theta / 2}\right) .
$$

Ensuite, on pose $X_{1}=\left(y, x, \delta, \eta, \nabla_{x} \xi, \tau\right)$ et on applique de nouveau la formule de Taylor au point $X_{1}$ à l'ordre 1 on aura

$$
\begin{aligned}
G(y, x, \delta, s, z)= & \delta^{-\theta} p\left(X_{1}\right)+s p_{t}^{\prime}\left(X_{1}\right)+\sum_{j=1}^{n-q}\left(c_{j}+s \tau_{x_{j}}^{\prime}\right) p_{\xi_{j}}^{\prime}\left(X_{1}\right) \\
& +\delta^{-\theta / 2} z p_{\tau}^{\prime}\left(X_{1}\right)+\frac{z^{2}}{2} p_{\tau \tau}^{\prime \prime}\left(X_{1}\right)+O\left(\delta^{\theta / 2}\right)
\end{aligned}
$$


Or d'après (4.5), $p\left(X_{1}\right)=p_{\tau}^{\prime}\left(X_{1}\right)=0$, d'où

$$
G(y, x, \delta, s, z)=s\left[p_{t}^{\prime}\left(X_{1}\right)+\sum_{j=1}^{n-q} \tau_{x_{j}}^{\prime} p_{\xi_{j}}^{\prime}\left(X_{1}\right)\right]+\sum_{j=1}^{n-q} c_{j} p_{\xi_{j}}^{\prime}\left(X_{1}\right)+\frac{z^{2}}{2} p_{\tau \tau}^{\prime \prime}\left(X_{1}\right)+O\left(\delta^{\theta / 2}\right) .
$$

Ainsi

$$
G\left(0,0,0,0, z_{0}\right)=\sum_{j=1}^{n-q} c_{j} p_{\xi_{j}}^{\prime}\left(0, \zeta_{0}\right)+\frac{z_{0}^{2}}{2} p_{\tau \tau}^{\prime \prime}\left(0, \zeta_{0}\right)
$$

On pose

$$
A=\sum_{j=1}^{n-q} c_{j} p_{\xi_{j}}^{\prime}\left(0, \zeta_{0}\right), \quad B=p_{t}^{\prime}\left(0, \zeta_{0}\right)+\sum_{j=1}^{n-q} \tau_{x_{j}}^{\prime} p_{\xi_{j}}^{\prime}\left(0, \zeta_{0}\right)
$$

Comme

$$
d_{\xi} p\left(0, \zeta_{0}\right) \neq 0
$$

on peut donc trouver $c \in \mathbb{R}^{n-q}$ tel que

$$
A=B \text {. }
$$

On déduit de (4.27), (4.32) que $z_{0}$ est donné par

$$
z_{0}^{2}=-\frac{2 B}{p_{\tau T}^{\prime \prime}\left(0, \zeta_{0}\right)} .
$$

D'après le lemme 4.2 et l'hypothèse de non stricte pseudoconvexité de l'hypersurface on a $z_{0}^{2}<0$. Ainsi $z_{0}$ est imaginaire pur. On choisit $z_{0}$ tel que $\operatorname{Im} z_{0}>0$. Donc d'après l'hypothèse (i) du théorème 3.1 on a

$$
\begin{gathered}
G\left(0,0,0,0, z_{0}\right)=0 \\
\frac{\partial G}{\partial z}\left(0,0,0,0, z_{0}\right)=z_{0} p_{\tau \tau}^{\prime \prime}\left(0, \zeta_{0}\right) \neq 0,
\end{gathered}
$$

d'après le théorème des fonctions implicites, il existe un voisinage $V$ de $(0,0,0,0)$ dans $\mathbb{R}^{q-1} \times \mathbb{R}^{n-q} \times \mathbb{R} \times \mathbb{R}$ et une fonction

$$
g: V \longrightarrow V\left(z_{0}\right) \subset \mathbb{C}, \quad(y, x, \delta, s) \longmapsto g(y, x, \delta, s)
$$

vérifiant

$$
\begin{aligned}
g(0,0,0,0) & =z_{0}, \\
G(y, x, \delta, s, g(y, x, \delta, s)) & =0 \text { dans } V .
\end{aligned}
$$

On pose

$$
D_{s} \varphi(y, x, \delta, s)=g(y, x, \delta, s)
$$

avec

$$
\varphi_{\mid s=0}=0,
$$


alors

$$
\frac{\partial^{2} \varphi}{\partial s^{2}}(y, x, \delta, s)=i \frac{\partial g}{\partial s}(y, x, \delta, s) .
$$

Par ailleurs, d'après (4.25), (4.39) et (4.41) on a

$$
p\left(y, x, \delta+\delta^{\theta} s, \eta, \nabla_{x} \tilde{\xi}+\delta^{\theta} s \nabla_{x} \tau, \tau+\delta^{\theta / 2} D_{s} \varphi(y, x, \delta, s)\right)=0,
$$

en dérivant cette expression par rapport à $s$ on obtient

$$
\delta^{\theta} p_{t}^{\prime}+\delta^{\theta} \tau_{x}^{\prime} \cdot p_{\xi}^{\prime}+\frac{\delta^{\theta / 2}}{i} \frac{\partial^{2} \varphi}{\partial s^{2}} p_{\tau}^{\prime}=0
$$

D'où en prenant $y=0, x=0, s=0$ et $\eta=\eta_{0}$ on obtient

$$
\begin{aligned}
\delta^{\theta} p_{t}^{\prime}\left(0,0, \delta, \eta_{0}, \nabla_{x} \tilde{\xi}, \tau+\delta^{\theta / 2} D_{s} \varphi\right) & \\
& +\delta^{\theta} \tau_{x}^{\prime}(0,0, \delta) \cdot p_{\xi}^{\prime}\left(0,0, \delta, \eta_{0}, \xi_{0}, \tau_{0}+\delta^{\theta / 2} D_{s} \varphi\right) \\
& +\frac{\delta^{\theta / 2}}{i} \frac{\partial^{2} \varphi}{\partial s^{2}}(0,0, \delta, 0) p_{\tau}^{\prime}\left(0,0, \delta, \eta_{0}, \xi_{0}, \tau_{0}+\delta^{\theta / 2} D_{s} \varphi\right)=0
\end{aligned}
$$

Appliquons la formule de Taylor à $p_{t}^{\prime}, p_{\xi}^{\prime}$ et $p_{\tau}^{\prime}$ à l'ordre 1 , au point

$$
X=\left(0,0, \delta, \eta_{0}, \nabla_{x} \xi(0,0, \delta), \tau(0,0, \delta)\right),
$$

il en résulte que

$$
\begin{aligned}
\delta^{\theta} p_{t}^{\prime}(X)+\delta^{\theta} \tau_{x}^{\prime}(0,0, \delta) & \cdot p_{\xi}^{\prime}(X)+\frac{\delta^{\theta / 2}}{i} \frac{\partial^{2} \varphi}{\partial s^{2}}(0,0, \delta, 0) p_{\tau}^{\prime}(X) \\
& +\frac{\delta^{\theta}}{i}\left(\frac{\partial^{2} \varphi}{\partial s^{2}} D_{s} \varphi\right)(0,0, \delta, 0) p_{\tau \tau}^{\prime \prime}(X)+o\left(\delta^{\theta}\right)=0 .
\end{aligned}
$$

D'après le lemme 4.1, nous avons pour $\delta<\delta_{0}$

$$
p_{\tau}^{\prime}\left(0,0, \delta, \eta_{0}, \nabla_{x} \xi(0,0, \delta), \tau(0,0, \delta)\right)=0
$$

d'où (4.48) devient

$$
\delta^{\theta} p_{t}^{\prime}(X)+\delta^{\theta} \tau_{x}^{\prime}(0,0, \delta) \cdot p_{\xi}^{\prime}(X)+\frac{\delta^{\theta}}{i}\left(\frac{\partial^{2} \varphi}{\partial s^{2}} D_{s} \varphi\right)(0,0, \delta, 0) p_{\tau \tau}^{\prime \prime}(X)+o\left(\delta^{\theta}\right)=0
$$

On en déduit que

$$
p_{t}^{\prime}(X)+\tau_{x}^{\prime}(0,0, \delta) \cdot p_{\xi}^{\prime}(X)+\frac{1}{i}\left(\frac{\partial^{2} \varphi}{\partial s^{2}} D_{s} \varphi\right)(0,0, \delta, 0) p_{\tau \tau}^{\prime \prime}(X)+o(1)=0 .
$$

En faisant tendre $\delta$ vers zéro, on obtient

$$
B+\frac{1}{i} \frac{\partial^{2} \varphi}{\partial s^{2}} z_{0} p_{\tau \tau}^{\prime \prime}\left(0, \zeta_{0}\right)=0,
$$


donc

$$
B+g_{s}^{\prime}(0,0,0,0) z_{0} p_{\tau \tau}^{\prime \prime}\left(0, \zeta_{0}\right)=0
$$

Par ailleurs, nous avons $z_{0}^{2}=-2 B / P_{\tau \tau}^{\prime \prime}\left(0, \zeta_{0}\right)$, ceci donne

$$
B z_{0}-2 B g_{s}^{\prime}(0)=0
$$

d'où $g_{s}^{\prime}(0)=z_{0} / 2$. Ainsi

$$
\operatorname{Re} \frac{\partial^{2} \varphi}{\partial s^{2}}(0)=-\operatorname{Im} g_{s}^{\prime}(0)=-\frac{1}{2} \operatorname{Im} z_{0}<0
$$

par suite $\beta(0,0,0,0)<0$ et

$$
\alpha(0,0,0)=\operatorname{Re} i g(0,0,0,0)=i z_{0}<0 .
$$

Corollaire 4.4. On $a$

$$
\begin{aligned}
p_{m}\left(y, x, \delta+\frac{s}{\lambda}, \eta, \nabla_{x} \tilde{\xi}+\frac{s}{\lambda} \nabla_{x} \tau, \tau+\frac{\lambda v}{\sigma^{1 / m_{n}}}\left(D_{s} \varphi+\frac{1}{v} D_{s}\right)\right) \\
=\frac{1}{\lambda v}\left[H(y, x, \delta, s) D_{s}+K(y, x, \delta, s)+\delta^{r} Q\left(y, x, \delta, s, D_{s}\right)\right],
\end{aligned}
$$

où $H, K$ et les coefficients de $Q$ sont réguliers, $H(0) \neq 0, r>0$ et $Q$ un opérateur différentiel en $D_{s}$.

Preuve. Fixons $\sigma^{1 / m_{n}}=\lambda^{3 / 2} v$. Dans cette preuve on écrira $p$ à la place de $p_{m}$. On a

$$
\begin{aligned}
p(y, x, \delta+ & \left.\frac{s}{\lambda}, \eta, \nabla_{x} \tilde{\xi}+\frac{s}{\lambda} \nabla_{x} \tau, \tau+\frac{\lambda v}{\sigma^{1 / m_{n}}}\left(D_{s} \varphi+\frac{1}{v} D_{s}\right)\right) \\
= & \sum_{\left|\alpha^{\prime}: m^{\prime}\right|+\left|\beta: m_{n}\right|+k / m_{n}=1} a_{\alpha^{\prime} \beta k} \eta^{\alpha^{\prime}}\left(\nabla_{x} \tilde{\xi}+\frac{s}{\lambda} \nabla_{x} \tau\right)^{\beta} \\
& \times\left(\tau+\delta^{\theta / 2}\left(D_{s} \varphi+\frac{1}{v} D_{s}\right)\right)^{k},
\end{aligned}
$$

où $\alpha^{\prime} \in \mathbb{N}^{q-1}, m^{\prime}=\left(m_{1}, \ldots, m_{q-1}\right), \beta \in \mathbb{N}^{n-q-1}$ et $k \in \mathbb{N}$.

Pour montrer qu'il existe $r>0$ tel que

$$
\begin{aligned}
{\left[\tau+\delta^{\theta / 2}\left(D_{s} \varphi+\frac{1}{v} D_{s}\right)\right]^{k}=} & \left(\tau+\delta^{\theta / 2} D_{s} \varphi\right)^{k}+k\left(\tau+\delta^{\theta / 2} D_{s} \varphi\right)^{k} \delta^{\theta / 2} D_{s} \\
& -a_{k} \tau^{k-2} \frac{\delta^{\theta}}{v} \varphi_{s s}^{\prime \prime}+\delta^{r} \frac{\delta^{\theta}}{v} R_{k}\left(y, x, \delta, s, D_{s}\right)
\end{aligned}
$$


il suffit de raisonner par récurrence sur $k$. De (4.59) on déduit que

$$
\begin{aligned}
p\left(y, x, \delta+\frac{s}{\lambda}, \eta, \nabla_{x} \tilde{\xi}+\frac{s}{\lambda} \nabla_{x} \tau, \tau+\delta^{\theta / 2}\left(D_{s} \varphi+\frac{1}{v} D_{s}\right)\right) \\
=\sum_{\left|\alpha^{\prime}: m^{\prime}\right|+\left|\beta: m_{n}\right|+k / m_{n}=1} a_{\alpha^{\prime} \beta k} \eta^{\alpha^{\prime}}\left(\nabla_{x} \tilde{\xi}+\frac{s}{\lambda} \nabla_{x} \tau\right)^{\beta}\left(\tau+\delta^{\theta / 2}\left(D_{s} \varphi\right)\right)^{k} \\
\quad+\sum_{\left|\alpha^{\prime}: m^{\prime}\right|+\left|\beta: m_{n}\right|+k / m_{n}=1} a_{\alpha^{\prime} \beta k} \eta^{\alpha^{\prime}}\left(\nabla_{x} \tilde{\xi}+\frac{s}{\lambda} \nabla_{x} \tau\right)^{\beta} k\left(\tau+\delta^{\theta / 2} D_{s} \varphi\right)^{k-1} \frac{\delta^{\theta / 2}}{v} D_{s} \\
\quad-\sum_{\left|\alpha^{\prime}: m^{\prime}\right|+\left|\beta: m_{n}\right|+k / m_{n}=1} a_{\alpha^{\prime} \beta k} \eta^{\alpha^{\prime}}\left(\nabla_{x} \tilde{\xi}+\frac{s}{\lambda} \nabla_{x} \tau\right)^{\beta} \tau^{k-2} \frac{\delta^{\theta}}{v} \varphi_{s s}^{\prime \prime} \\
\quad+\delta^{r} \sum_{\left|\alpha^{\prime}: m^{\prime}\right|+\left|\beta: m_{n}\right|+k / m_{n}=1} \frac{\delta^{\theta}}{v} a_{\alpha^{\prime} \beta k} \eta^{\alpha^{\prime}}\left(\nabla_{x} \tilde{\xi}+\frac{s}{\lambda} \nabla_{x} \tau\right)^{\beta} R_{k}\left(y, x, \delta, s, D_{s}\right) .
\end{aligned}
$$

Or d'après le lemme 4.3 et ce qui précède on a

$$
\begin{aligned}
& \sum_{\left|\alpha^{\prime}: m^{\prime}\right|+\left|\beta: m_{n}\right|+k / m_{n}=1} a_{\alpha^{\prime} \beta k} \eta^{\alpha^{\prime}}\left(\nabla_{x} \tilde{\xi}+\frac{s}{\lambda} \nabla_{x} \tau\right)^{\beta}\left(\tau+\delta^{\theta / 2}\left(D_{s} \varphi\right)\right)^{k} \\
& =p\left(y, x, \delta+\frac{s}{\lambda}, \eta, \nabla_{x} \tilde{\xi}+\frac{s}{\lambda} \nabla_{x} \tau, \tau+\delta^{\theta / 2} D_{s} \varphi\right)=0 \\
& \sum_{\left|\alpha^{\prime}: m^{\prime}\right|+\left|\beta: m_{n}\right|+k / m_{n}=1} a_{\alpha^{\prime} \beta k} \eta^{\alpha^{\prime}}\left(\nabla_{x} \tilde{\xi}+\frac{s}{\lambda} \nabla_{x} \tau\right)^{\beta} k\left(\tau+\delta^{\theta / 2} D_{s} \varphi\right)^{k-1} \frac{\delta^{\theta / 2}}{\nu} D_{s} \\
& =p_{\tau}^{\prime}\left(y, x, \delta+\frac{s}{\lambda}, \eta, \nabla_{x} \tilde{\xi}+\frac{s}{\lambda} \nabla_{x} \tau, \tau+\delta^{\theta / 2} D_{s} \varphi\right) \frac{\delta^{\theta / 2}}{\nu} D_{s}, \\
& -\sum_{\left|\alpha^{\prime}: m^{\prime}\right|+\left|\beta: m_{n}\right|+k / m_{n}=1} a_{\alpha^{\prime} \beta k} \eta^{\alpha^{\prime}}\left(\nabla_{x} \tilde{\xi}+\frac{s}{\lambda} \nabla_{x} \tau\right)^{\beta} \tau^{k-2} \frac{\delta^{\theta}}{v} \varphi_{s s}^{\prime \prime} \\
& =\frac{\delta^{\theta}}{v} K(y, x, \delta, s), \\
& \delta^{r} \sum_{\left|\alpha^{\prime}: m^{\prime}\right|+\left|\beta: m_{n}\right|+k / m_{n}=1} \frac{\delta^{\theta}}{\nu} a_{\alpha^{\prime} \beta k} \eta^{\alpha^{\prime}}\left(\nabla_{x} \tilde{\xi}+\frac{s}{\lambda} \nabla_{x} \tau\right)^{\beta} R_{k}\left(y, x, \delta, s, D_{s}\right) \\
& =\delta^{r} \frac{\delta^{\theta}}{v} Q\left(y, x, \delta, s, D_{s}\right) .
\end{aligned}
$$

Reprenons le terme $\sum_{\left|\alpha^{\prime}: m^{\prime}\right|+\left|\beta: m_{n}\right|+k / m_{n}=1} a_{\alpha^{\prime} \beta k} \eta^{\alpha^{\prime}}\left(\nabla_{x} \tilde{\xi}+(s / \lambda) \nabla_{x} \tau\right)^{\beta} k(\tau+$ $\left.\delta^{\theta / 2} D_{s} \varphi\right)^{k-1}\left(\delta^{\theta / 2} / \nu\right) D_{s}$ et appliquons la formule de Taylor d'abord au point $X=$ $\left(y, x, \delta+s / \lambda, \eta, \nabla_{x} \tilde{\xi}+(s / \lambda) \nabla_{x} \tau, \tau\right)$ à l'ordre 2, puis au point $X_{1}=\left(y, x, \delta, \eta, \nabla_{x} \xi, \tau\right)$ à l'ordre 1 , nous obtenons

$$
\begin{aligned}
p_{\tau}^{\prime}(y, x, \delta+ & \left.\frac{s}{\lambda}, \eta, \nabla_{x} \tilde{\xi}+\frac{s}{\lambda} \nabla_{x} \tau, \tau+\delta^{\theta / 2} D_{s} \varphi\right) \\
& =p_{\tau}^{\prime}\left(X_{1}\right)+\delta^{\theta / 2}\left(D_{s} \varphi\right) p_{\tau \tau}^{\prime \prime}\left(X_{1}\right)+O\left(\delta^{\theta}\right) .
\end{aligned}
$$


Il en résulte qu'il existe $r>0$ tel que

$$
\begin{aligned}
& p\left(y, x, \delta+\frac{s}{\lambda}, \eta, \nabla_{x} \tilde{\xi}+\frac{s}{\lambda} \nabla_{x} \tau, \tau+\delta^{\theta / 2}\left(D_{s} \varphi+\frac{1}{v} D_{s}\right)\right) \\
& =\frac{1}{\lambda \nu}\left[p_{\tau \tau}^{\prime \prime}\left(y, x, \delta, \eta, \nabla_{x} \xi, \tau\right)\left(D_{s} \varphi\right) D_{s}+K(y, x, \delta, s)+\delta^{r} Q\left(y, x, \delta, D_{s}\right)\right] .
\end{aligned}
$$

Nous avons par hypothèse (i) du théorème 3.1 que $p_{\tau \tau}^{\prime \prime}\left(0,0,0, \zeta_{0}\right) \neq 0$. En posant

$$
H(y, x, \delta, s)=p_{\tau \tau}^{\prime \prime}\left(y, x, \delta, \eta, \nabla_{x} \xi, \tau\right)\left(D_{s} \varphi\right),
$$

nous obtenons le résultat cherché.

4.3. Recollement des solutions asymptotiques. Les constructions précédentes nous donnent une famille de fonctions $u_{\delta}$ dépendant du paramètre $\delta>0$ qui pour $t$ près de $\delta$ vérifient $P u_{\delta}=0$.

Dans la suite, on donne à $\delta$ la suite de valeurs $\delta_{k}=b_{k}=k^{-\rho}$ avec $k \in \mathbb{N}^{*}$ et $\rho>0$. On note $v=v_{k}=k^{\varepsilon}$ avec $\varepsilon>0$ et $\sigma=\sigma_{k}=\left(\lambda_{k}^{3 / 2} v_{k}\right)^{m_{n}}$. On notera pour $t \in\left[b_{k+1}, b_{k-1}\right]$, $t=\delta_{k}+s / \lambda_{k}$, la fonction

$$
\begin{aligned}
u_{k}\left(y, x, \delta_{k}+\frac{s}{\lambda_{k}}\right)= & e^{i\left(\sum_{j=1}^{q-1} \sigma^{1 / m_{j}} \eta_{j} y_{j}+\sigma^{1 / m_{n}}\left(\tilde{\xi}\left(y, x, \delta_{k}\right)+\left(s / \lambda_{k}\right) \tau\left(y, x, \delta_{k}\right)\right)\right)} \\
& \times e^{v_{k} \varphi\left(y, x, \delta_{k}, s\right)} e^{-\gamma\left(y, x, \delta_{k}\right)} w\left(y, x, \delta_{k}, s\right) .
\end{aligned}
$$

On a pour $k$ assez grand et $t \in\left[b_{k+1}, b_{k-1}\right]$

$$
|s|=\lambda_{k}\left|t-\delta_{k}\right| \leq \lambda_{k}\left|\delta_{k-1}-\delta_{k}\right| \simeq \rho k^{\rho(\theta-1)-1} .
$$

Notons (c.1) la condition suivante :

(c.1) $\rho(\theta-1)-1<0$.

Si (c.1) est vérifiée, la fonction $u_{k}$ est bien définie.

L'opérateur $\tilde{P}$ défini par (4.4) s'écrit alors

$$
\begin{aligned}
\tilde{P}= & \sigma P_{m}\left(y, x, \delta+\frac{s}{\lambda},\left(\eta_{j}+\sigma^{1 / m_{n}-1 / m_{j}}\left(\nabla_{j} \tilde{\xi}+\frac{s}{\lambda} \nabla_{j} \tau\right)+\frac{v}{i} \frac{1}{\sigma^{1 / m_{j}}} \nabla_{j} \varphi\right.\right. \\
& \left.\quad-\frac{1}{i} \frac{1}{\sigma^{1 / m_{j}}} \nabla_{j} \gamma+\frac{1}{\sigma^{1 / m_{j}}} D_{y_{j}}\right)_{j \leq q-1} ; \nabla_{x} \tilde{\xi}+\frac{s}{\lambda} \nabla_{x} \tau \\
& \left.+\frac{v}{i} \frac{1}{\sigma^{1 / m_{n}}} \nabla_{x} \varphi-\frac{1}{i} \frac{1}{\sigma^{1 / m_{n}}} \nabla_{x} \gamma+\frac{1}{\sigma^{1 / m_{n}}} D_{x}, \tau+\frac{\lambda \nu}{\sigma^{1 / m_{n}}}\left(D_{s} \varphi+D_{s}\right)\right) \\
& +\sigma^{\kappa}(\cdots)+\cdots
\end{aligned}
$$

avec $\kappa<1$.

On impose que la fonction $\gamma$ et les paramètres $\lambda, \sigma, \delta$ vérifient les conditions suivantes :

(c.2) $\sigma^{-1 / m_{j}} \nabla_{j} \gamma$ bornée pour $\delta$ voisin de zéro,

(c.3) $\sigma^{-1 / m_{n}} \nabla_{x} \gamma$ bornée pour $\delta$ voisin de zéro,

(c.4) $\lambda^{-1} \sigma^{1 / m_{n}}-1 / m_{j}$ bornée pour $\delta$ voisin de zéro. 
4.3.1. Choix de $\gamma$. On va déterminer la fonction $\gamma(y, x, \delta)$ de sorte qu'on puisse "recoller" les fonctions $u_{k}$. Pour cela on choisira les fonctions $\gamma\left(y, x, \delta_{k}\right)$ de sorte que $\left|u_{k}\right| \gg\left|u_{k+1}\right|$ près de $\delta_{k} ;\left|u_{k+1}\right| \gg\left|u_{k}\right|$ près de $\delta_{k+1}$ et $\left|u_{k}\right|=\left|u_{k+1}\right|$ en un point proche du milieu de $\left[\delta_{k}, \delta_{k+1}\right]$.

Pour obtenir ce résultat on a besoin du lemme suivant.

LEMME 4.5. Posons pour $t \in\left[\delta_{k+1}, \delta_{k-1}\right]$

$$
\begin{aligned}
G_{k}(y, x, t)= & v_{k} \operatorname{Re} \varphi\left(y, x, \delta_{k}, s_{k}\right) \\
& -v_{k+1} \operatorname{Re} \varphi\left(y, x, \delta_{k+1}, s_{k+1}\right), \quad s_{j}=\lambda_{j}\left(t-\delta_{j}\right) .
\end{aligned}
$$

Notons $t_{k}=\delta_{k} / 3+(2 / 3) \delta_{k+1}$ et $I_{k}(y, x)=G_{k}\left(y, x, t_{k}\right)$, alors

$$
I_{k}(y, x) \simeq-\alpha(y, x, 0) \frac{\rho}{3} k^{\rho(\theta-1)+\varepsilon-1}, \quad \text { quand } k \longrightarrow+\infty .
$$

Preuve. On a $s_{k}=\lambda_{k}\left(t-\delta_{k}\right)$. On pose $l_{k}=(1 / 3)\left(\delta_{k}-\delta_{k+1}\right)$. Alors $l_{k} \simeq(\rho / 3) k^{-\rho-1}$ pour $k$ assez grand. Ainsi pour $t=t_{k}=\delta_{k}-2 l_{k}=\delta_{k+1}+l_{k}$ on a $s_{k}=\lambda_{k}\left(t_{k}-\delta_{k}\right)=$ $-2 \lambda_{k} l_{k}$ et $s_{k+1}=\lambda_{k+1} l_{k}$. On a donc

$$
\begin{aligned}
& v_{k} \operatorname{Re} \varphi\left(y, x, \delta_{k}, s_{k}\right)-v_{k+1} \operatorname{Re} \varphi\left(y, x, \delta_{k+1}, s_{k+1}\right) \\
&=k^{\varepsilon}\left[\alpha\left(y, x, \delta_{k}\right) s_{k}+\beta\left(y, x, \delta_{k}, s_{k}\right) s_{k}^{2}\right] \\
&-(k+1)^{\varepsilon}\left[\alpha\left(y, x, \delta_{k+1}\right) \cdot s_{k+1}+\beta\left(y, x, \delta_{k+1}, s_{k+1}\right) \cdot s_{k+1}^{2}\right] \\
&= k^{\varepsilon}\left[\alpha\left(y, x, \delta_{k}\right)\left(-2 k^{\rho \theta} l_{k}+\beta\left(y, x, \delta_{k}, s_{k}\right)\left(4 k^{2 \rho \theta} l_{k}^{2}\right)\right)\right] \\
&-(k+1)^{\varepsilon}\left[\alpha\left(y, x, \delta_{k+1}\right)(k+1)^{\rho \theta} l_{k}+\beta\left(y, x, \delta_{k+1}, s_{k+1}\right) l_{k}^{2}(k+1)^{2 \rho \theta}\right] \\
&=-\frac{\rho}{3}\left[2 \alpha\left(y, x, \delta_{k}\right) k^{\varepsilon+\rho \theta-\rho-1}+\alpha\left(y, x, \delta_{k+1}\right)(k+1)^{\varepsilon+\rho \theta} k^{-\rho-1}\right] \\
&+l_{k}^{2}\left[4 \beta_{k} k^{2 \rho \theta+\varepsilon}-\beta_{k+1}(k+1)^{2 \rho \theta+\varepsilon}\right] .
\end{aligned}
$$

Notons $E_{1}=-(\rho / 3)\left[2 \alpha\left(y, x, \delta_{k}\right) k^{\varepsilon+\rho \theta-\rho-1}+\alpha\left(y, x, \delta_{k+1}\right)(k+1)^{\varepsilon+\rho \theta} k^{-\rho-1}\right]$ et $E_{2}=l_{k}^{2}\left[4 \beta_{k} k^{2 \rho \theta+\varepsilon}-\beta_{k+1}(k+1)^{2 \rho \theta+\varepsilon}\right]$.

On commence par calculer $E_{1}$. On a pour $k$ assez grand

$$
\alpha\left(y, x, \delta_{k}\right)=\alpha\left(y, x, k^{-\rho}\right)=\alpha(y, x, 0)+O\left(k^{-\rho}\right),
$$

d'où

$$
\begin{aligned}
E_{1}=k^{\varepsilon+\rho \theta-\rho-1}[ & -\frac{2 \rho}{3} \alpha(y, x, 0)+O\left(k^{-\rho}\right) \\
& \left.-\frac{\rho}{3} \alpha(y, x, 0)\left(1+\frac{1}{k}\right)^{\varepsilon+\rho \theta}+O\left((k+1)^{-\rho}\right)\right],
\end{aligned}
$$

par suite

$$
E_{1} \simeq-k^{\varepsilon+\rho \theta-\rho-1} \rho \alpha(y, x, 0)
$$


quand $k$ tend vers l'infini. L'expression $E_{2}$ s'écrit aussi sous la forme

$$
E_{2}=l_{k}^{2}\left[4 \beta_{k} k^{2 \rho \theta+\varepsilon}-\beta_{k+1}(k+1)^{2 \rho \theta+\varepsilon}\right]
$$

or on a

$$
l_{k}^{2} \simeq \frac{\rho^{2}}{9} k^{-2 \rho-2}
$$

quand $k$ tend vers $+\infty$ et

$$
\beta\left(y, x, s_{k}, \delta_{k}\right)=\beta\left(y, x, s_{k}, k^{-\rho}\right)=\beta\left(y, x, s_{k}, 0\right)+O\left(k^{-\rho}\right) .
$$

On a

$$
\begin{aligned}
s_{k} & =\lambda_{k}\left(t_{k}-\delta_{k}\right)=\delta_{k}^{-\theta}\left[\frac{1}{3} \delta_{k}+\frac{2}{3} \delta_{k+1}-\delta_{k}\right] \\
& =\frac{2}{3} \delta_{k}^{-\theta}\left[\delta_{k+1}-\delta_{k}\right]=-2 \delta_{k}^{-\theta} l_{k} \simeq-\frac{2}{3} \rho k^{\rho \theta-\rho-1}, \quad \text { quand } k \rightarrow+\infty
\end{aligned}
$$

D'où, quand $k$ tend vers l'infini on a

$$
s_{k} \simeq-\frac{2}{3} \rho k^{\rho \theta-\rho-1}, \quad s_{k+1} \simeq \frac{1}{3} \rho k^{\rho \theta-\rho-1} .
$$

Ainsi on a

$$
\beta_{k}=\beta\left(y, x, s_{k}, \delta_{k}\right)=\beta(y, x, 0,0)+O\left(k^{\rho \theta-\rho-1}\right)+O\left(k^{-\rho}\right) .
$$

On en déduit que

$$
E_{2} \simeq \beta(y, x, 0,0) \frac{\rho^{2}}{3} k^{2(\rho(\theta-1)-1)+\varepsilon},
$$

quand $k \rightarrow+\infty$. Par suite on a

$$
E_{1}+E_{2} \simeq-\rho \alpha(y, x, 0) k^{\varepsilon+\rho(\theta-1)-1}, \quad \text { quand } k \longrightarrow+\infty .
$$

On obtient ainsi le résultat du lemme 4.5.

Démontrons maintenant le résultat énoncé au début du paragraphe 4.3.1. On pose pour $k_{0}$ assez grand

$$
\gamma_{k}(y, x)=-\sum_{j=k_{0}}^{k-1} I_{j}(y, x),
$$

on a

$$
\left(\gamma_{k+1}-\gamma_{k}\right)(y, x)=-I_{k}(y, x)
$$

D’après (4.69), on a

$$
I_{k}(y, x) \simeq-\alpha(y, x, 0) \rho k^{\rho(\theta-1)+\varepsilon-1},
$$

le signe de l'expression $\rho(\theta-1)+\varepsilon-1$ nous sera imposé ultérieurement par la condition (c.7). Ainsi on a

$$
I_{k}(y, x) \simeq-\int_{k}^{k+1} \alpha(y, x, 0) \rho u^{\varepsilon+\rho \theta-\rho-1} d u .
$$


On peut donc écrire

$$
\gamma_{k}(y, x) \simeq \int_{k_{0}}^{k} \alpha(y, x, 0) \rho u^{\varepsilon+\rho \theta-\rho-1} d u,
$$

d'où

$$
\begin{aligned}
\gamma_{k}(y, x) & \simeq \frac{\rho k^{\varepsilon+\rho(\theta-1)}}{(\varepsilon+\rho(\theta-1))} \alpha(y, x, 0) \\
& \simeq \frac{\rho \delta^{-(\theta-1+\varepsilon / \rho)}}{(\varepsilon+\rho(\theta-1))} \alpha(y, x, 0) .
\end{aligned}
$$

Vérifions (c.2), (c.3) et (c.4). On a $\sigma^{1 / m_{j}}=\left(\lambda_{k}^{3 / 2} v_{k}\right)^{m_{n} / m_{j}}$, d'où

$$
\sigma=\left(k^{3 / 2 \rho \theta} k^{\varepsilon}\right)^{m_{n}}, \quad \sigma^{1 / m_{j}}=\left(k^{\varepsilon+(3 / 2) \rho \theta}\right)^{m_{n} / m_{j}} .
$$

Posons $\Gamma_{j}=m_{n} / m_{j}>1$, alors

$$
\begin{gathered}
\sigma^{-1 / m_{j}} \nabla_{y} \gamma_{k}(y, x) \simeq \frac{\rho}{(\varepsilon+\rho(\theta-1))} \nabla_{y} \alpha(y, x, 0) \delta^{-\left((\theta-1+\varepsilon / \rho)-\Gamma_{j}((3 / 2) \theta+\varepsilon / \rho)\right)}, \\
\theta-1+\frac{\varepsilon}{\rho}-\Gamma_{j}\left(\frac{3}{2} \theta+\frac{\varepsilon}{\rho}\right)=\theta\left(1-\frac{3 \Gamma_{j}}{2}\right)+\frac{\varepsilon}{\rho}\left(1-\Gamma_{j}\right)-1<0 .
\end{gathered}
$$

On a aussi

$$
\begin{aligned}
\sigma^{-1 / m_{n}} \nabla_{x} \gamma\left(y, x, \delta_{k}\right) & \simeq \nabla_{x} \alpha(y, x, 0) \frac{\rho}{\varepsilon+\rho(\theta-1)} \delta^{-((\theta-1+\varepsilon / \rho)-(3 / 2) \theta-\varepsilon / \rho)} \\
& \simeq \nabla_{x} \alpha(y, x, 0) \frac{\rho}{\varepsilon+\rho(\theta-1)} \delta^{\theta / 2+1} \\
\lambda_{k}^{-1} \sigma_{k}^{1 / m_{n}-1 / m_{j}} & \simeq k^{\rho \theta\left(-(3 / 2) \Gamma_{j}+1 / 2\right)} k^{\varepsilon\left(1-\Gamma_{j}\right)}
\end{aligned}
$$

On remarque que le second membre de cette dernière équivalence tend vers 0 quand $k$ tend vers $+\infty$. Ainsi les conditions (c.2), (c.3) et (c.4) sont vérifiées. On choisira $\theta$ et $\rho$ de telle sorte que la condition (c.1) est vérifiée.

\subsection{2. Équation de transport et choix de $w$}

LEMME 4.6. On $a$

$$
\frac{1}{\sigma} \tilde{P}=\frac{1}{\lambda \nu}\left[H(y, x, \delta, s) D_{s}+K(y, x, \delta, s)+\delta^{\tilde{r}} \tilde{Q}\left(y, x, \delta, s, D_{y}, D_{x}, D_{s}\right)\right]
$$

où $H, K$ et les coefficients de $\tilde{Q}$ sont réguliers, $H(0,0,0,0) \neq 0, \tilde{r}>0$, à condition que l'on ait

(c.5) $\varepsilon\left[1-m_{n}(1-\kappa)\right]<\rho \theta\left[3 m_{n}(1-\kappa) / 2-1\right]$ pour tout $\kappa, 0 \leq \kappa \leq 1-1 / m_{n}$ et

(c.6) $\varepsilon<\rho \theta / 2 ; \varepsilon<\rho(1-\theta / 2)$. 
Preuve. Nous avons

$$
\begin{aligned}
\sigma^{-1} \tilde{P}=P_{m}\left(y, x, \delta+\frac{s}{\lambda},\right. & \left(\eta_{j}+\sigma^{1 / m_{n}-1 / m_{j}}\left(\nabla_{j} \tilde{\xi}+\frac{s}{\lambda} \nabla_{j} \tau\right)+\frac{v}{i} \frac{1}{\sigma^{1 / m_{j}}} \nabla_{j} \varphi\right. \\
& \left.-\frac{1}{i} \frac{1}{\sigma^{1 / m_{j}}} \nabla_{j} \gamma+\frac{1}{\sigma^{1 / m_{j}}} D_{y_{j}}\right)_{j \leq q-1} ; \nabla_{x} \tilde{\xi}+\frac{s}{\lambda} \nabla_{x} \tau \\
& \left.+\frac{v}{i} \frac{1}{\sigma^{1 / m_{n}}} \nabla_{x} \varphi-\frac{1}{i} \frac{1}{\sigma^{1 / m_{n}}} \nabla_{x} \gamma+\frac{1}{\sigma^{1 / m_{n}}} D_{x}, \tau+\frac{\lambda \nu}{\sigma^{1 / m_{n}}}\left(D_{s} \varphi+D_{s}\right)\right) \\
& +\sigma^{\kappa-1}(\ldots)+\cdots
\end{aligned}
$$

avec $\kappa<1$. En utilisant un développement de Taylor de $P_{m}$ à l'ordre 1 au point $X=$ $\left(y, x, \delta+s / \lambda, \eta, \nabla_{x} \tilde{\xi}+(s / \lambda) \nabla_{x} \tau, \tau+\left(\lambda \nu / \sigma^{1 / m_{n}}\right)\left(D_{s} \varphi+D_{s}\right)\right)$, on remarque d'après le corollaire 4.4 que $\sigma^{-1} \tilde{P}$ s'écrit sous la forme (4.91) si les conditions suivantes sont satisfaites :

(i) $\sigma^{\kappa-1}=o(1 / \lambda v)$,

(ii) $\delta^{-d} \sigma^{-1 / m_{n}}=o(1 / \lambda \nu), \delta^{-d} \sigma^{-1 / m_{j}}=o(1 / \lambda \nu)$ avec $d=\theta-1+\varepsilon / \rho$,

(iii) $v \sigma^{-1 / m_{n}}=o(1 / \lambda \nu), v \sigma^{-1 / m_{j}}=o(1 / \lambda \nu)$.

La condition (i) est vraie d'après (c.5), ainsi les termes de $\sigma^{-1} \tilde{P}$ provenant des termes d'ordre inférieurs de $P$ sont inclus dans $\delta^{\tilde{r}} \tilde{Q}$. La condition (ii) est vraie d'après la deuxième partie de (c.6). La condition (iii) est vraie d'après la première partie de (c.6).

Déterminons la suite de fonctions $w_{j}(y, x, \delta, s), j \geq 0$, par

$$
\begin{gathered}
\left(H D_{s}+K\right) w_{0}=0, \quad w_{0}(y, x, \delta, 0)=1, \\
\left(H D_{s}+K\right) w_{j}=-\tilde{Q} w_{j-1}, \quad w_{j}(y, x, \delta, 0)=0, \quad j \geq 1 .
\end{gathered}
$$

Ainsi la solution formelle $\tilde{w}=\sum_{j \geq 0} w_{j} \delta^{j \tilde{r}}$ sera alors la solution de

$$
\left(H D_{s}+K+\delta^{\tilde{r}} \tilde{Q}\right) \tilde{w}=0 .
$$

On prend une fonction de $(y, x, \delta, s, z), Z(y, x, \delta, s, z), C^{\infty}$ près de $(0,0,0,0)$, telle que

$$
Z \sim \sum_{j \geq 0} w_{j} z^{j}
$$

quand $z$ tend vers 0 , au sens suivant :

$$
\forall k \in \mathbb{N}, \forall \alpha \in \mathbb{N}^{n-q}, \forall \beta \in \mathbb{N}^{q-1}, \forall N \in \mathbb{N}, \quad \exists c_{k \alpha \beta N} \geq 0,
$$

tel que pour $(y, x, \delta, s, z)$ près de l'origine on a

$$
\left|D_{s}^{k} D_{x}^{\alpha} D_{y}^{\beta}\left(Z-\sum_{j=0}^{N} w_{j} z^{j}\right)\right| \leq c_{k \alpha \beta N}|z|^{N+1} .
$$

Si on prend $w(y, x, \delta, s)=Z\left(y, x, \delta, s, \delta^{\tilde{r}}\right)$, on obtient $\left(H D_{s}+K+\delta^{\tilde{r}} \tilde{Q}\right) w=o\left(\delta^{\infty}\right)$. 
LEMme 4.7. Pour tout $t \in\left[\delta_{k+1}, \delta_{k-1}\right] ;(y, x) \in V$ où $V$ est un voisinage de $(0,0)$, soit

$$
\begin{aligned}
u_{k}(y, x, t)= & e^{i \sum_{j=1}^{q-1} \sigma^{1 / m_{j}} \eta_{j} y_{j}+\sigma^{1 / m_{n}}\left(\tilde{\xi}\left(y, x, \delta_{k}\right)+\left(t-\delta_{k}\right) \tau\left(y, x, \delta_{k}\right)\right)} \\
& \times e^{v_{k} \varphi\left(y, x, \delta_{k}, s\right)} e^{-\gamma\left(y, x, \delta_{k}\right)} w\left(y, x, \delta_{k}, s\right)
\end{aligned}
$$

avec $s=\lambda_{k}\left(t-\delta_{k}\right)$ et soit $f_{k}=P u_{k} / u_{k}$. Alors il existe $k_{0}>0$ tel qu'on a

$$
\begin{gathered}
\forall(\beta, \alpha, l) \in \mathbb{N}^{q-1} \times \mathbb{N}^{n-q} \times \mathbb{N}, \forall N \in \mathbb{N}, N \text { assez grand, } \exists c_{\alpha \beta l N}>0 ; \exists \Gamma_{N}>0 \\
\text { tels que }\left|D_{y}^{\beta} D_{x}^{\alpha} D_{t}^{l} f_{k}(y, x, t)\right| \leq c_{\alpha \beta l N} k^{-\Gamma_{N}}
\end{gathered}
$$

pour tout $k, k \geq k_{0}$ et $(y, x, t) \in V \times\left[\delta_{k+1}, \delta_{k-1}\right]$.

Preuve. On a $P u_{k} / u_{k}=\tilde{P} w_{k} / w_{k}=(\sigma / \lambda v)\left(1 / w_{k}\right)\left[\left(H D_{s}+K+\delta^{\tilde{r}} \tilde{Q}\right) w_{k}\right]$. Or

$$
w_{k}=\sum_{j=0}^{N-1}\left(\delta^{\tilde{r}}\right)^{j} w_{j}\left(y, x, s, \delta_{k}\right)+\left(\delta^{\tilde{r}}\right)^{N} R_{N}\left(y, x, s, \delta_{k}\right)
$$

avec $R_{N}$ fonction de classe $C^{\infty}$ et bornée dans un voisinage de zéro. D’où

$$
\begin{aligned}
f_{k}=\frac{\sigma}{\lambda v} \frac{1}{w_{k}}\left[\sum_{j=0}^{N-1}\left(\delta_{k}^{\tilde{r}}\right)^{j}\left(H D_{s}+K\right) w_{j}\right. \\
\left.\quad \quad+\left(\delta_{k}^{\tilde{r}}\right)^{N}\left(H D_{s}+K\right) R_{N}+\sum_{j=0}^{N-1}\left(\delta_{k}^{\tilde{r}}\right)^{j} \tilde{Q} w_{j}+\left(\delta_{k}^{\tilde{r}}\right)^{N+1} \tilde{Q} R_{N}\right] \\
=\frac{\sigma}{\lambda v} \frac{1}{w_{k}}\left[\left(H D_{s}+K\right) w_{0}+\left(\delta_{k}^{\tilde{r}}\right)^{N} \tilde{Q} w_{N-1}+\left(\delta^{\tilde{r}}\right)^{N}\left(H D_{s}+K \delta^{\tilde{r}} \tilde{Q}\right) R_{N}\right] \\
=\frac{\left(k^{3 \rho \theta / 2+\varepsilon}\right)^{m_{n}}}{k^{\rho \theta+\varepsilon}} \frac{1}{w_{k}} k^{-\rho \tilde{r} N}\left[\tilde{Q} w_{N-1}+\left(H D_{s}+K+\delta^{\tilde{r}} \tilde{Q}\right) R_{N}\right] \\
=k^{\rho \theta\left((3 / 2) m_{n}-1\right)+\varepsilon\left(m_{n}-1\right) \rho \tilde{r} N} \frac{1}{w_{k}}\left[\tilde{Q} w_{N-1}+\left(H D_{s}+K+\delta^{\tilde{r}} \tilde{Q}\right) R_{N}\right] \\
=k^{\rho\left[-\tilde{r} N+\theta\left((3 / 2) m_{n}-1\right)\right]+\varepsilon\left(m_{n}-1\right)} \frac{1}{w_{k}}\left[\tilde{Q} w_{N-1}+\left(H D_{s}+K+\delta^{\tilde{r}} \tilde{Q}\right) R_{N}\right] \\
=k^{-\rho\left[\tilde{r} N-\theta\left((3 / 2) m_{n}-1\right)\right]+\varepsilon\left(m_{n}-1\right)} \frac{1}{w_{k}}\left[\tilde{Q} w_{N-1}+\left(H D_{s}+K+\delta^{\tilde{r}} \tilde{Q}\right) R_{N}\right] .
\end{aligned}
$$

On en déduit que $\left|f_{k}\right| \leq C_{N} k^{-\Gamma_{N}}$. On procède de même pour les dérivées de $f_{k}$.

4.3.3. Étude de l'ensemble où $\left|u_{k}\right|=\left|u_{k+1}\right|$. Pour $(y, x, t) \in V \times\left[\delta_{k+1}, \delta_{k-1}\right], V$ est un voisinage de $(0,0) \subset \mathbb{R}^{q-1} \times \mathbb{R}^{n-q}$ posons

$$
F_{k}(y, x, t)=\log \left|\frac{u_{k}}{u_{k+1}}\right| .
$$

LEMME 4.8. Il existe des constantes c et $\eta$ strictement positives telles qu'on ait

$$
\frac{\partial F_{k}}{\partial t} \geq c k^{\eta}
$$

avec 
(c.7) $\varepsilon+\rho(\theta-1)-1>0, \eta=2 \rho \theta-\rho+\varepsilon-1$.

Preuve. On a

$$
\begin{aligned}
\frac{\partial F_{k}}{\partial t}= & {\left[\lambda_{k} v_{k} \frac{\partial \operatorname{Re} \varphi}{\partial s}\left(y, x, s_{k}, \delta_{k}\right)-\lambda_{k+1} v_{k+1} \frac{\partial \operatorname{Re} \varphi}{\partial s}\left(y, x, s_{k+1}, \delta_{k+1}\right)\right] } \\
& +\left[\lambda_{k} \frac{w_{s}^{\prime}\left(y, x, s_{k}, \delta_{k}\right)}{w\left(y, x, s_{k}, \delta_{k}\right)}-\lambda_{k+1} \frac{w_{s}^{\prime}\left(y, x, s_{k+1}, \delta_{k+1}\right)}{w\left(y, x, s_{k+1}, \delta_{k+1}\right)}\right],
\end{aligned}
$$

On note $E_{3}=\left[\lambda_{k}\left(w_{s}^{\prime}\left(y, x, s_{k}, \delta_{k}\right) / w\left(y, x, s_{k}, \delta_{k}\right)\right)-\lambda_{k+1}\left(w_{s}^{\prime}\left(y, x, s_{k+1}, \delta_{k+1}\right) /\right.\right.$ $\left.\left.w\left(y, x, s_{k+1}, \delta_{k+1}\right)\right)\right]$.

on écrira

$$
\frac{\partial F_{k}}{\partial t}=\left[\lambda_{k} v_{k} \frac{\partial \operatorname{Re} \varphi}{\partial s}\left(y, x, s_{k}, \delta_{k}\right)-\lambda_{k+1} v_{k+1} \frac{\partial \operatorname{Re} \varphi}{\partial s}\left(y, x, s_{k+1}, \delta_{k+1}\right)\right]+E_{3} .
$$

D'après (4.25) on a

$$
\operatorname{Re} \varphi(y, x, s, \delta)=\alpha(y, x, \delta) s+\beta(y, x, \delta, s) s^{2},
$$

donc

$$
\begin{aligned}
\frac{\partial \operatorname{Re} \varphi}{\partial s} & =\alpha(y, x, \delta)+2 \beta(y, x, \delta, s) s+\beta_{s}^{\prime}(y, x, \delta, s) s^{2} \\
& =\alpha(y, x, \delta)+s\left(2 \beta(y, x, \delta, s)+\beta_{s}^{\prime}(y, x, \delta, s) s\right) \\
& =\alpha(y, x, \delta)+\beta_{1}(y, x, \delta, s) s,
\end{aligned}
$$

où $\beta_{1}(y, x, \delta, s)=2 \beta(y, x, \delta, s)+\beta_{s}^{\prime}(y, x, \delta, s) s$, avec $\beta_{1}(0,0,0,0)<0$. On a

$$
\begin{aligned}
\frac{\partial F_{k}}{\partial t} & \\
= & {\left[\left(\lambda_{k} v_{k}-\lambda_{k+1} v_{k+1}\right) \alpha\left(y, x, \delta_{k}\right)\right] } \\
& +\left[\lambda_{k+1} v_{k+1}\left(\alpha\left(y, x, \delta_{k}\right)-\alpha\left(y, x, \delta_{k+1}\right)\right)\right] \\
& +\left[-\beta_{1}\left(y, x, \delta_{k+1}, s_{k+1}\right) \frac{\left(t-\delta_{k+1}\right)}{\delta_{k+1}^{2 \theta}} v_{k+1}+\beta_{1}\left(y, x, \delta_{k}, s_{k}\right) \frac{\left(t-\delta_{k}\right)}{\delta_{k}^{2 \theta}} v_{k}\right]+E_{3} \\
= & {\left[\left(\lambda_{k} v_{k}-\lambda_{k+1} v_{k+1}\right) \alpha\left(y, x, \delta_{k}\right)\right]+\left[\lambda_{k+1} v_{k+1}\left(\alpha\left(y, x, \delta_{k}\right)-\alpha\left(y, x, \delta_{k+1}\right)\right)\right] } \\
& +\left[-\beta_{1}\left(y, x, \delta_{k+1}, s_{k+1}\right)\left(\frac{\left(t-\delta_{k+1}\right)}{\delta_{k+1}^{2 \theta}}\right) v_{k+1}+\beta_{1}\left(y, x, \delta_{k}, s_{k}\right)\left(\frac{\left(t-\delta_{k}\right)}{\delta_{k}^{2 \theta}}\right) v_{k}\right]+E_{3} .
\end{aligned}
$$

Notons $E_{4}=\left[\left(\lambda_{k} v_{k}-\lambda_{k+1} v_{k+1}\right) \alpha\left(y, x, \delta_{k}\right)\right], E_{5}=\left[\lambda_{k+1} v_{k+1}\left(\alpha\left(y, x, \delta_{k}\right)-\alpha(y\right.\right.$, $\left.\left.\left.x, \delta_{k+1}\right)\right)\right]$ et $E_{6}=\left[-\beta_{1}\left(y, x, \delta_{k+1}, s_{k+1}\right)\left(\left(t-\delta_{k+1}\right) / \delta_{k+1}^{2 \theta}\right) v_{k+1}+\beta_{1}\left(y, x, \delta_{k}, s_{k}\right)\right.$ $\left.\left(\left(t-\delta_{k}\right) / \delta_{k}^{2 \theta}\right) v_{k}\right]$

Évaluons $E_{4}$, nous avons

$$
\left|E_{4}\right| \leq c_{0}\left|\lambda_{k}-\lambda_{k+1}\right| \leq c_{1} k^{\rho \theta+\varepsilon-1}, \quad \text { avec } c_{0}>0, c_{1}>0 .
$$

La fonction $\alpha(y, x, \delta)$ étant de classe $C^{\infty}$ par rapport à $\left(y, x, \delta^{r}\right)$ avec $r>0$, alors il existe des constantes $c^{\prime}>0$ et $c^{\prime \prime}>0$ telles que

$$
\left|\alpha\left(y, x, \delta_{k}\right)-\alpha\left(y, x, \delta_{k+1}\right)\right| \leq c^{\prime}\left(\delta_{k}^{r}-\delta_{k+1}^{r}\right) \leq c^{\prime \prime} k^{-\rho r-1} .
$$


On en déduit qu' il existe une constante $c_{2}>0$ telle que $\left|E_{5}\right| \leq c_{2} k^{\rho \theta+\varepsilon-\rho r-1}$. Estimons $E_{6}$,

$$
\begin{aligned}
E_{6}= & -\beta_{1}\left(y, x, \delta_{k+1}, s_{k+1}\right) \\
& \times\left[\left(\frac{t-\delta_{k+1}}{\delta_{k+1}^{2 \theta}}-\frac{t-\delta_{k}}{\delta_{k+1}^{2 \theta}}\right) v_{k+1}+\left(t-\delta_{k}\right)\left(\frac{v_{k+1}}{\delta_{k+1}^{2 \theta}}-\frac{v_{k}}{\delta_{k}^{2 \theta}}\right)\right] \\
& -\frac{v_{k}}{\delta_{k}^{2 \theta}}\left(t-\delta_{k}\right)\left[\beta_{1}\left(y, x, s_{k+1}, \delta_{k+1}\right)-\beta_{1}\left(y, x, s_{k}, \delta_{k}\right)\right], \\
= & -\beta_{1}\left(y, x, s_{k+1}, \delta_{k+1}\right)\left[\frac{\delta_{k}-\delta_{k+1}}{\delta_{k+1}^{2 \theta}} v_{k+1}\right] \\
& -\beta_{1}\left(y, x, s_{k+1}, \delta_{k+1}\right)\left[\frac{v_{k+1}}{\delta_{k+1}^{2 \theta}}-\frac{v_{k}}{\delta_{k}^{2 \theta}}\right]\left(t-\delta_{k}\right) \\
& -\frac{v_{k}}{\delta_{k}^{2 \theta}}\left(t-\delta_{k}\right)\left[\beta_{1}\left(y, x, s_{k+1}, \delta_{k+1}\right)-\beta_{1}\left(y, x, s_{k}, \delta_{k}\right)\right] .
\end{aligned}
$$

Pour $k$ assez grand on a $\left(\delta_{k}-\delta_{k+1}\right) / \delta_{k+1}^{2 \theta} \simeq \rho k^{2 \theta-\rho-1}$ et $v_{k+1} \simeq k^{\varepsilon}$. Comme $\beta_{1}(0,0,0,0)<$ 0 il existe donc une constante $\tilde{c}_{0}>0$ telle que

$$
\begin{aligned}
-\beta_{1}\left(y, x, s_{k+1}, \delta_{k+1}\right)\left[\frac{\delta_{k}-\delta_{k+1}}{\delta_{k+1}^{2 \theta}} v_{k+1}\right] & =-\beta_{1}\left(y, x, s_{k+1}, \delta_{k+1}\right)\left[\frac{\delta_{k}-\delta_{k+1}}{\delta_{k+1}^{2 \theta}} v_{k+1}\right] \\
& \geq \tilde{c}_{0} k^{2 \rho \theta-\rho-1+\varepsilon} .
\end{aligned}
$$

On a $v_{k} / \delta_{k}^{2 \theta} \simeq k^{2 \rho \theta+\varepsilon}$, d'où on a $v_{k+1} / \delta_{k+1}^{2 \theta}-v_{k} / \delta_{k}^{2 \theta} \simeq(2 \rho \theta+\varepsilon) k^{2 \rho \theta+\varepsilon-1}$. Comme $t \in$ $\left[\delta_{k+1}, \delta_{k-1}\right]$, on a $\left|t-\delta_{k}\right| \leq c^{t e} \rho k^{-\rho-1}$. On en déduit qu'il existe une constante $\tilde{c}_{1}>0$ telle que

$$
\begin{aligned}
& \left|-\beta_{1}\left(y, x, s_{k+1}, \delta_{k+1}\right)\left[\frac{v_{k+1}}{\delta_{k+1}^{2 \theta}}-\frac{v_{k}}{\delta_{k}^{2 \theta}}\right]\left(t-\delta_{k}\right)\right| \\
& =\left|\left(t-\delta_{k}\right) \beta_{1}\left(y, x, s_{k+1}, \delta_{k+1}\right)\left[\frac{v_{k+1}}{\delta_{k+1}^{2 \theta}}-\frac{v_{k}}{\delta_{k}^{2 \theta}}\right]\right| \\
& \leq \tilde{c}_{1} k^{2 \rho \theta+\varepsilon-2-\rho} .
\end{aligned}
$$

On a

$$
\begin{aligned}
-\frac{v_{k}}{\delta_{k}^{2 \theta}}(t & \left.-\delta_{k}\right)\left[\beta_{1}\left(y, x, s_{k+1}, \delta_{k+1}\right)-\beta_{1}\left(y, x, s_{k}, \delta_{k}\right)\right] \\
& =\frac{v_{k}}{\delta_{k}^{2 \theta}}\left(t-\delta_{k}\right)\left[\beta_{1}\left(y, x, s_{k+1}, \delta_{k+1}\right)-\beta_{1}\left(y, x, s_{k}, \delta_{k}\right)\right] \\
& =o\left(k^{2 \rho \theta-\rho-1+\varepsilon}\right) .
\end{aligned}
$$

Ainsi on déduit qu'il existe une constante $c_{2}>0$ telle que $E_{6} \geq c_{3} k^{2 \rho \theta-\rho-1+\varepsilon}$ On voit facilement qu'il existe une constante $c_{4}>0$ telle que $\left|E_{3}\right| \leq c_{4} k^{\rho \theta}$. On a donc obtenu 
les inégalités suivantes :

$$
\begin{aligned}
\left|E_{4}\right| & \leq c_{0}\left|\lambda_{k}-\lambda_{k+1}\right| \leq c_{1} k^{\rho \theta+\varepsilon-1}, \quad \text { avec } c_{0}>0, c_{1}>0 \\
\left|E_{5}\right| & \leq c_{2} k^{\rho \theta+\varepsilon-\rho r-1}, \quad \text { avec } c_{2}>0 \\
E_{6} & \geq c_{3} k^{2 \rho \theta-\rho-1+\varepsilon}, \quad \text { avec } c_{3}>0 \\
\left|E_{3}\right| & \leq c_{4} k^{\rho \theta}, \quad \text { avec } c_{4}>0
\end{aligned}
$$

On a

$$
\begin{gathered}
\rho \theta+\varepsilon-1 \leq 2 \rho \theta-\rho-1+\varepsilon \quad(\operatorname{car} \theta>1 \text { et } \rho>0), \\
\rho \theta+\varepsilon-\rho r-1 \leq 2 \rho \theta-\rho-1+\varepsilon \quad(\operatorname{car} r>0, \rho>0 \text { et } \theta>1), \\
\rho \theta<2 \rho \theta-\rho-1+\varepsilon \Longleftrightarrow \rho(\theta-1)-1+\varepsilon>0 \quad \text { (d' après (c.7)). }
\end{gathered}
$$

On en déduit qu'il existe une constante $c>0$ telle que pour $k$ assez grand

$$
\frac{\partial F_{k}}{\partial t}(y, x, t) \geq c k^{\eta}, \quad \text { avec } \eta=2 \rho \theta-\rho-1+\varepsilon
$$

d'où le lemme 4.8 .

Estimons maintenant la fonction $F_{k}$. D'après les expressions (4.68), (4.98) et (4.102) on peut écrire

$$
\begin{aligned}
F_{k}(y, x, t)= & \gamma\left(y, x, \delta_{k+1}\right)-\gamma\left(y, x, \delta_{k}\right)+G_{k}(y, x, t) \\
& +\log \left(\left|\frac{w\left(y, x, \delta_{k}, \lambda_{k}\left(t-\delta_{k}\right)\right)}{w\left(y, x, \delta_{k+1}, \lambda_{k+1}\left(t-\delta_{k+1}\right)\right)}\right|\right) .
\end{aligned}
$$

En utilisant (4.87) et en prenant $t=t_{k}=(1 / 3) \delta_{k}+(2 / 3) \delta_{k+1}$, on obtient

$$
\begin{aligned}
F_{k}\left(y, x, t_{k}\right)= & \gamma\left(y, x, \delta_{k+1}\right)-\gamma\left(y, x, \delta_{k}\right)+I_{k}(y, x) \\
& +\log \left(\left|\frac{w\left(y, x, \delta_{k}, \lambda_{k}\left(t_{k}-\delta_{k}\right)\right)}{w\left(y, x, \delta_{k+1}, \lambda_{k+1}\left(t_{k}-\delta_{k+1}\right)\right)}\right|\right) \\
= & \log \left(\left|\frac{w\left(y, x, \delta_{k}, \lambda_{k}\left(t_{k}-\delta_{k}\right)\right)}{w\left(y, x, \delta_{k+1}, \lambda_{k+1}\left(t_{k}-\delta_{k+1}\right)\right)}\right|\right) \\
= & O(1) .
\end{aligned}
$$

Ainsi $F_{k}\left(y, x, t_{k}\right)$ est bornée indépendemment de $k$. Pour que $k^{\eta} l_{k}$ tende vers $+\infty$ quand $k$ tend vers $+\infty$ il faut que

(c.8) $2 \rho \theta+\varepsilon-2(\rho+1)>0$.

D'après (4.103), on a $F_{k}(y, x, t)$ s'annule en un point d'un intervalle contenant $t_{k}$. D'après le théorème des fonctions implicites, il existe une fonction $t_{k}(y, x)$ de classe $C^{\infty}$ sur un voisinage $V_{0}$ de $(0,0)$ à valeurs dans un intervalle ouvert de $\left[\delta_{k+1}, \delta_{k}\right]$, telle que $F_{k}\left(y, x, t_{k}(y, x)\right)=0$. Par ailleurs, on vérifie que $t_{k}(y, x)=t_{k}+e_{k}(y, x)$, avec $e_{k}(y, x)=O\left(k^{-\eta}\right)$. 
Les étapes suivantes étant standard, les démonstrations sont les mêmes que dans [1, 3]. On ne mentionnera que les étapes, laissant le lecteur se rapporter à [3] pour les détails.

4.3.4. Modification des $u_{k}$. On va modifier légèrement les fonctions $u_{k}$ de manière à pouvoir bien définir la perturbation $a$.

On construit une suite de fonctions, $y_{k}(y, x, s)$, nulles sur les surfaces $t=t_{k}(y, x)$ et $t=t_{k-1}(y, x)$, plus petites que toute puissance de $1 / k$ pour $t \in\left[\delta_{k+1}, \delta_{k-1}\right]$, telles que, en notant

$$
v_{k}(y, x, t)=u_{k}(y, x, t)\left(1+y_{k}(y, x, s)\right),
$$

on a

(1) $\tilde{F}_{k}=\log \left(\left|v_{k} / v_{k+1}\right|\right)$ satisfait (4.103) et s'annule pour $t=t_{k}(y, x)$,

(2) $g_{k}=P v_{k} / v_{k}$ satisfait (4.99),

(3) $g_{k}$ est plate sur $\left\{(y, x, t): t=t_{k}(y, x)\right\}$ et $\left\{(y, x, t): t=t_{k-1}(y, x)\right\}$.

Cette construction utilise uniquement le lemme 4.7 et le fait que les surfaces $t=c^{\text {te }}$ sont non caractéristiques pour $P$.

4.4. Dernière étape. On choisit $\chi$ une fonction de classe $C^{\infty}$ sur $\mathbb{R}$ à support compact telle que

$$
\chi(s)=1 \quad \text { pour }|s| \leq \frac{3}{4}, \quad \operatorname{supp} \chi \subset[-1,1], \quad 0 \leq \chi \leq 1 .
$$

On pose $\chi_{k}(t)=\chi\left(\left(t-\delta_{k}\right) / 3 l_{k}\right)$ et $u(y, x, t)=\sum_{k \geq k_{0}} \chi_{k}(t) v_{k}(y, x, t), k_{0}$ est un entier assez grand. On vérifie alors que $a=-P u / u$ est une fonction de classe $C^{\infty}$ sur un voisinage de zéro, plate sur $t=0$.

Il reste à vérifier que $u$ est plate sur $t=0$. Or, ce résultat découle du fait que d'après l'expression (4.87), la fonction $\gamma_{k}(y, x)$ est $O\left(k^{\rho(\theta-1)+\varepsilon}\right)$ et $\left|v_{k} \operatorname{Re} \varphi(y, x, t)\right| \leq$ $C k^{\rho(\theta-1)+\varepsilon-1}$.

4.5. Compatibilité des conditions (c.1),...,(c.8) et choix effectif des paramètres. Par hypothèse nous avons $\theta>1, \varepsilon>0, \rho>0$ et $K \leq\left(1-1 / m_{n}\right)$. Les conditions sont

(c.1) $\rho(\theta-1)-1<0$,

(c.5) $\varepsilon\left[1-m_{n}(1-K)\right]<\rho \theta\left[3 m_{n}(1-K) / 2-1\right]$,

(c.6) $\varepsilon<\rho \theta / 2$ et $\varepsilon<\rho(1-\theta / 2)$,

(c.7) $\varepsilon+\rho(\theta-1)-1>0$,

(c.8) $2 \rho \theta+\varepsilon-2(\rho+1)>0$,

fixons $\theta=9 / 8, \varepsilon=3 / 4$ et choisissons $\rho=6$. Ainsi les conditions (c.1), (c.2), (c.3), (c.4), (c.5), (c.6), (c.7) et (c.8) sont vérifiées. Ceci achève la démonstration du théorème 3.1.

\section{BIBLIOGRAPHIE}

[1] S. Alinhac, Non-unicité du problème de Cauchy pour des opérateurs de type principal, Sémin. Goulaouic-Meyer-Schwartz, Equations Deriv. Partielles 1980-1981, Exposé no. 16, École Polytechnique, Paris, 1981, pp. 1-8 (French).

[2] L. Hörmander, Non-uniqueness for the Cauchy problem, Fourier Integral Operators and Partial Differential Equations (Colloq. Internat., Université de Nice, Nice, 1974), Lecture Notes in Mathematics, vol. 459, Springer, Berlin, 1975, pp. 36-72. 
[3] R. Lascar and C. Zuily, Unicité et non unicité du problème de Cauchy pour une classe d'opérateurs différentiels à caractéristiques doubles, Duke Math. J. 49 (1982), no. 1, 137162.

[4] A. Pliś, A smooth linear elliptic differential equation without any solution in a sphere, Comm. Pure Appl. Math. 14 (1961), 599-617.

Khalgui-Ounaïes Hella : Département de Mathématiques, Faculté des Sciences de TUNis, CAMPus UniVERsitaire, 1060 Tunis, TUNisia

E-mail address: he11a.kha1gui@fst.rnu.tn 


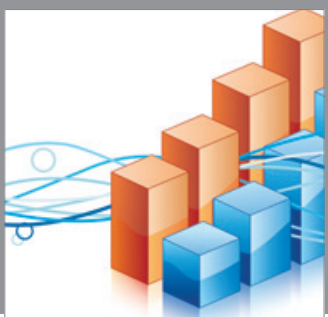

Advances in

Operations Research

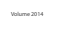

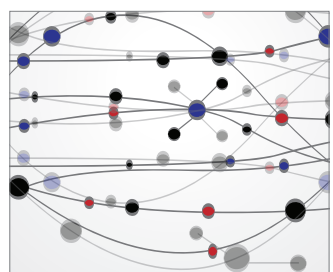

\section{The Scientific} World Journal
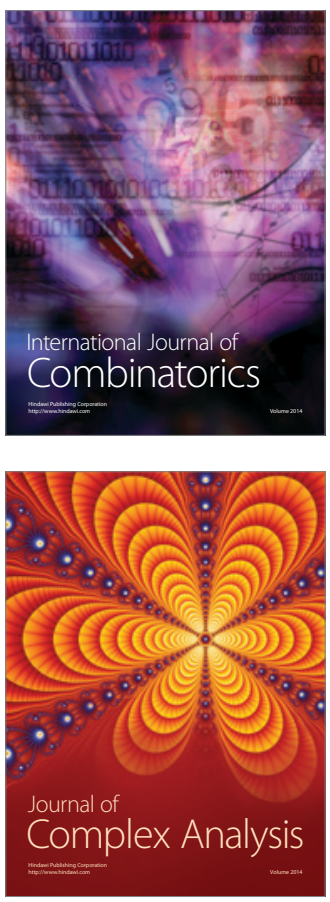

International Journal of

Mathematics and

Mathematical

Sciences
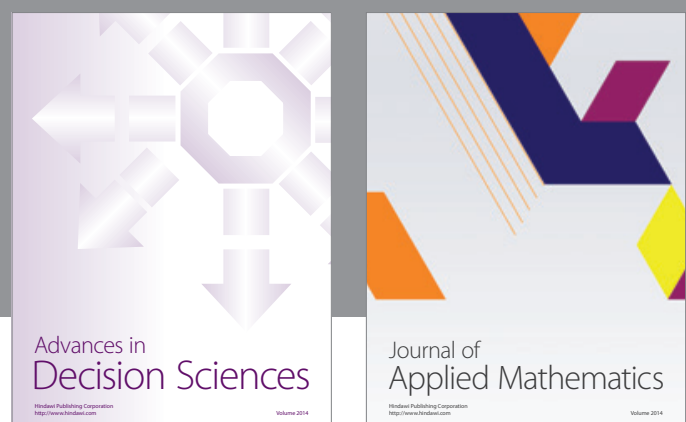

Journal of

Applied Mathematics
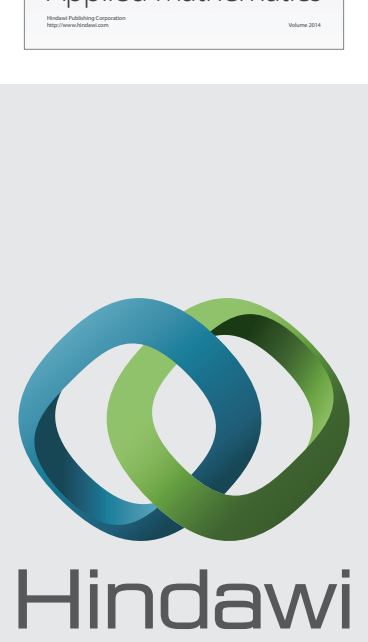

Submit your manuscripts at http://www.hindawi.com
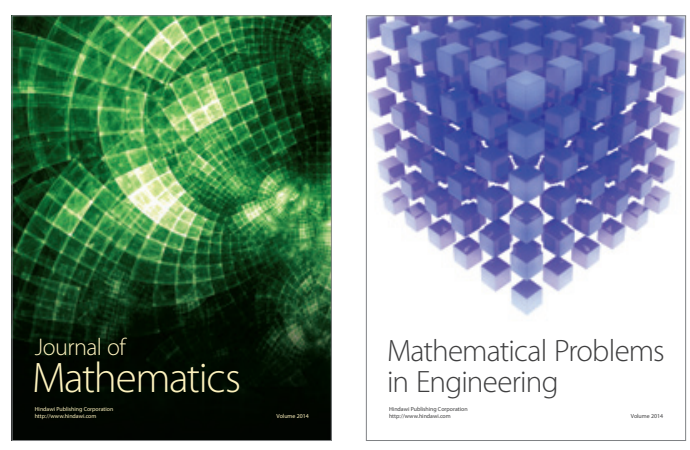

Mathematical Problems in Engineering
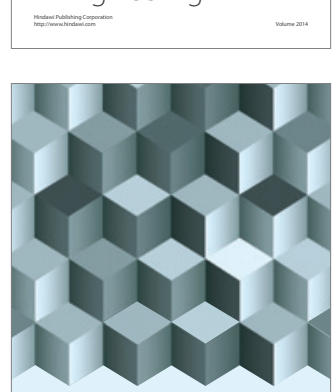

Journal of

Function Spaces
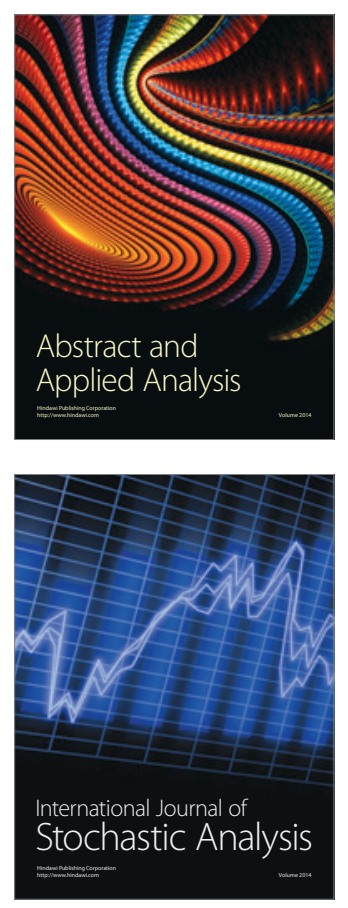

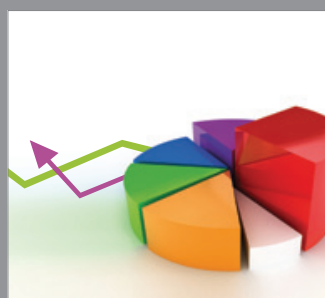

ournal of

Probability and Statistics

Promensencen
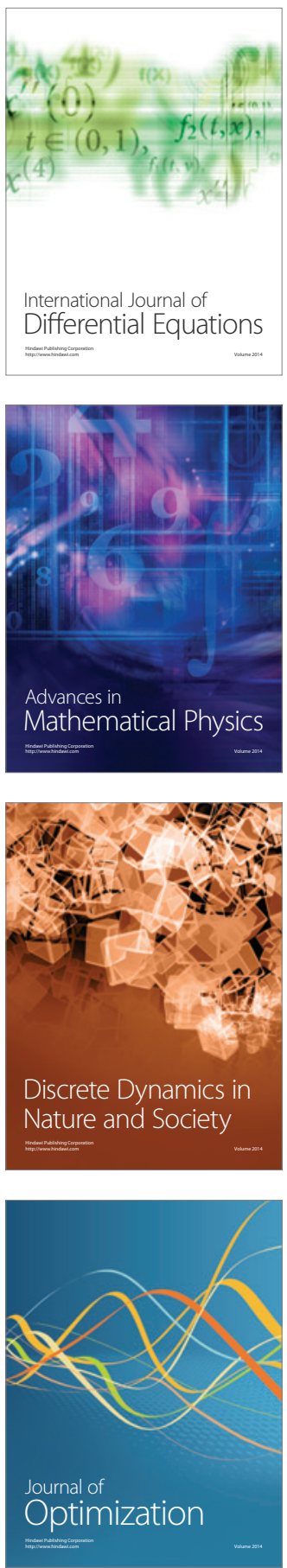